\title{
A novel method for producing solid polymer microneedles using laser ablated moulds in an injection moulding process
}

\author{
Tim Evens ${ }^{1}$, Olivier Malek ${ }^{2}$, Sylvie Castagne ${ }^{3}$, D. Seveno ${ }^{4}$, Albert Van Bael ${ }^{1,4}$ \\ ${ }^{1}$ KU Leuven, Diepenbeek Campus, Materials Technology TC, Wetenschapspark 27, 3590 Diepenbeek, \\ Belgium
}

${ }^{2}$ Sirris, Precision Manufacturing, Wetenschapspark 9, 3590 Diepenbeek, Belgium

${ }^{3} \mathrm{KU}$ Leuven, Department of Mechanical Engineering and and Flanders Make@KU Leuven-MaPS, Celestijnenlaan 300, 3001 Leuven, Belgium, Member Flanders Make

${ }^{4} \mathrm{KU}$ Leuven, Department of Materials Engineering, Kasteelpark Arenberg 44, 3001 Leuven, Belgium

Correspondence to: Tim Evens (E-mail: tim.evens@kuleuven.be)

\section{ABSTRACT}

Microneedle arrays are minimally invasive devices which offer a pain free, straightforward and efficient method for transdermal drug delivery. Manufacturing these micro-systems represents a real challenge, and a mass production process with high volume outputs at low cost is lacking. In this study, we present a novel method to produce polymer microneedles using laser ablated moulds in an injection moulding process. We successfully created cone-shaped micro holes with low tip radii in a tool steel mould, using a femtosecond laser with a cross-hatching strategy. Finally, the attained mould was used in an injection moulding process to replicate polypropylene microneedles.

\section{KEYWORDS}

Laser machining; Injection Moulding; Microneedles; Micro Manufacturing 


\section{INTRODUCTION}

Microneedles are microscale invasive devices, usually arranged in an array, designed to by-pass the human stratum corneum skin barrier [1]-[3]. The length of the needles can range from $50 \mu \mathrm{m}-2000$ $\mu \mathrm{m}$ and when penetrated in the skin, microscopic aqueous pores are created, through which drugs can diffuse to the dermal microcirculation [4]. The advantages of these devices have already extensively been studied in medical research [5]-[8]. At this stage, a production process to manufacture these microneedles in large volumes at a low cost is required [2], [9]. A promising manufacturing process which fits these criteria is polymer injection moulding. By applying the negative geometry of the microneedles on a mould cavity, millions of polymer microneedle systems can be produced at a low cost [10]. However, within the current state of the art, methods for applying the negative of the microneedles on the injection mould are limited to expensive and time consuming precision techniques such as micro-electrical discharge machining ( $\mu$-EDM) [11]-[14], milling [15], drilling [16], forging [17] or indirect mould making [18]. Besides, manufacturing microneedle cavities with a sharp tip has proven to be very difficult with these techniques, while small tip-radii are essential for microneedles to effectively penetrate the skin [9], [11].

In this study, a novel manufacturing process to produce sharp-tipped solid polymer microneedle arrays using laser ablated moulds in an injection moulding process is presented. 


\section{MATERIALS AND METHODS}

\subsection{Materials}

The thermoplastic material is a high melt flow polypropylene (PP) grade 515A from SABIC, with a melt flow rate of $24 \mathrm{~g} / 10 \mathrm{~min}\left(230^{\circ} \mathrm{C} / 2,16 \mathrm{~kg}\right)$. A low-corrosion tool steel (grade 1.2083 - AISI 420), was selected for the mould insert material.

\subsection{Representation of the process chain}

A micromachining system with a laser source is employed to laser ablate a mould insert with dimensions $70 \mathrm{~mm} \times 60 \mathrm{~mm} \times 4.4 \mathrm{~mm}$ (Figure 1a). The insert is then placed in the mould (Figure 1b). Afterwards, the mould is used in an injection moulding process (Figure 1c) to replicate polymer microneedles (Figure 1d).

a) Laser machining

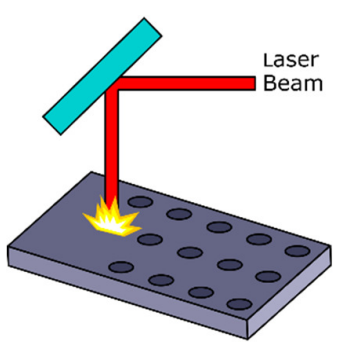

b) Removable mould insert

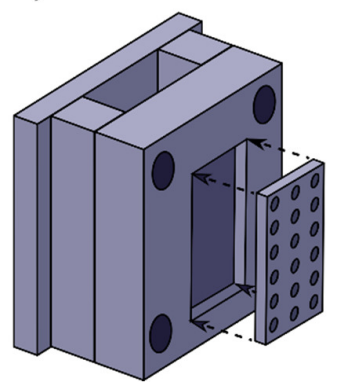

c) Injection moulding

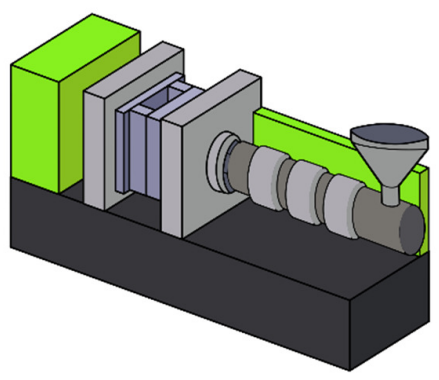

d) Polymer microneedles

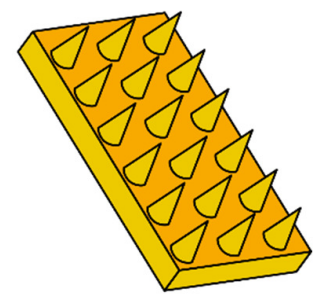

FIGURE 1 Illustration of the process chain to produce solid polymer microneedles.

\subsection{Laser machining}

A micromachining system (Lasea LS5) with a femtosecond laser (Satsuma HP, Amplitude Systemes) is employed to ablate the mould insert. The laser source emits a beam with a wavelength of $1030 \mathrm{~nm}$, a pulse repetition rate of $500 \mathrm{kHz}$ and a pulse length of $250 \mathrm{fs}$. The maximum average power of the laser is $7.85 \mathrm{~W}$, giving a deliverable pulse energy of $15.7 \mu \mathrm{J}$. Within the focal plane, the laser beam is steered at a scanning speed of $100 \mathrm{~mm} / \mathrm{s}$ with a spot size of $15.0 \mu \mathrm{m}$ and a pulse fluence of 2.22 $\mathrm{J} / \mathrm{cm}^{2}$.

A cross-hatching strategy was implemented as illustrated in Figure 2. The laser spot is scanned in parallel lines within a circular shape, in perpendicular directions. The distance between two consecutive lines is defined as the hatch pitch. Once the laser has scanned one circular grid, the focal point is lowered (with a vertical distance defined as the layer pitch) and the laser scans the same grid. This process is repeated multiple times and defines the number of layers The selected laser parameters in this work are based on a previous exploratory research [19]. A hatch pitch of $2 \mu \mathrm{m}, \mathrm{a}$ layer pitch of $15 \mu \mathrm{m}$, a base diameter of $300 \mu \mathrm{m}$ and a number of layers varying between 150,300 , 
450 and 600 were selected. For each set of parameters, holes were created in an array of $3 \times 3$ with a distance of $300 \mu \mathrm{m}$ between the centre points.

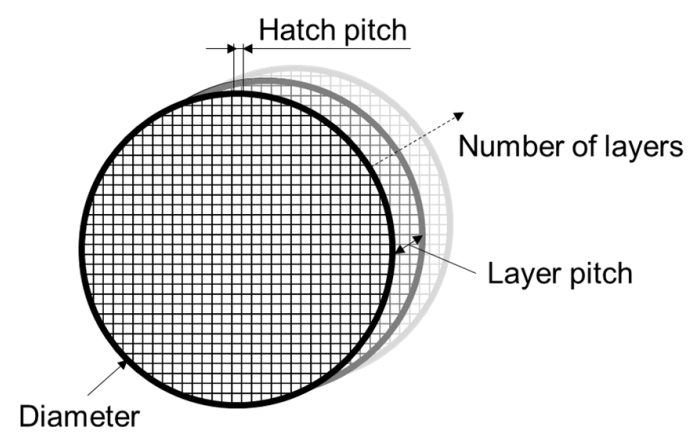

FIGURE 2 Illustration of the cross-hatching laser strategy.

\subsection{Replication of microneedles through injection moulding}

Injection moulding was done on an Engel ES 200/35 HL hydraulic injection moulding machine. The mould temperature is controlled by a Wittmann Tempro-controller. The injection moulded specimen is a $50 \mathrm{~mm} \times 60 \times 1.5 \mathrm{~mm}$ flat plate. Injection moulding parameters were defined to achieve a high replication fidelity. The injection temperature was set to $240^{\circ} \mathrm{C}$. The mould temperature was set to $80^{\circ} \mathrm{C}$, the maximum recommended ejection temperature of polypropylene [20]. The volumetric injection rate was set to a high value of $128 \mathrm{~cm}^{3} / \mathrm{s}$ to reduce the viscosity during injection. The holding pressure was set to a value just below the occurrence of flash, corresponding to 475 bar.

\subsection{Topography characterization}

$\mu$-CT was used to characterize the geometry of the ablated needle cavities. The $\mu$-CT system (Phoenix Nanotom) is equipped with a nanofocus X-ray tube. A molybdenum target was chosen for the high X-ray absorbing steel samples. The mould insert was cut down in cubic samples with an edge length of $3 \mathrm{~mm}$. For each scan, $2400 \mathrm{X}$-ray 2D projection images were obtained. Acquisition parameters were fixed for all samples as follows: voltage $=100 \mathrm{kV}$, current $=158 \mathrm{~A}$, voxel size $=3.75$ $\mu \mathrm{m}$, and a $0.1 \mathrm{~mm}$ copper and $0.1 \mathrm{~mm}$ aluminium filter were used during scanning. Reconstruction of the acquired 2D projections into 3D volumes was performed using GE Phoenix datos $\mid x$ REC software. Reconstructed XY datasetswere exported for further analysis and visualization within Fiji ImageJ.

The geometries of the replicated thermoplastic microneedles were assessed using a digital microscope (Keyence VH-S30) with a maximum magnification of X200. 


\section{RESULTS AND DISCUSSION}

The analysis of the reconstructed $\mu \mathrm{CT} X Y$ datasets of the laser ablated tool steel with different layers (Fig. 3) clearly shows cone-shaped micro holes with very sharp tip radii. It can be observed that the hole depth is increased when a higher number of layers is used, due to the increase in accumulated fluence. The dimension of the acquired tip radii is close to the voxel size of the CT measurement of $3.75 \mu \mathrm{m}$. This dimension is much lower than micro holes with tip radii of $32 \mu \mathrm{m}$ up to $80 \mu \mathrm{m}$, obtained respectively by [14] and [13]. The obtained cone-shape is expected to be a result of the plasma shielding effect [21]. During the ablation process, vapour material is partially ejected from the micro cavity, but a part of it remains near the surface, held by surface tension forces, and forms a recast layer after each pulse [22]. Additionally, the vaporised material forms plasma during the duration of each pulse. This plasma absorbs and defocuses the laser beam, creating a protective shield called "plasma shielding effect". The deeper the ablated micro hole, the more difficult the vaporised material can escape the hole, resulting in an expanding plasma which in turn increases the protective shield [23]. Therefore, using the proposed laser strategy, it is possible to create coneshaped micro holes with variable hole depths.

The laser ablated mould insert was used in an injection moulding process to replicate the micro holes. The replicated polymer microneedles (Fig. 4) show a similar cone-shape as the corresponding micro holes. However, by comparing the depth of the micro holes to the length of the polymer microneedles, it is observed that the micro holes were not completely replicated in the injection moulding process. As a result of the incomplete filling, the tip radii of the microneedles $(<20 \mu \mathrm{m})$ are larger than the very small tip radii of the micro holes. To give an estimation of volumetric filling, 2D profiles of both the micro holes and microneedles were imported in a CAD programme. By assuming that the profiles are axisymmetric, corresponding volumes could be compared. The percentages of volumetric filling for the micro holes created with laser parameters for 150, 300, 450 and 600 layers are respectively $97 \%, 94 \%, 88 \%$ and $85 \%$. Besides, a higher percentage of volumetric filling corresponds to a smaller tip radius. This radius is, as already indicated, the most important feature of the microneedle. The smaller the tip radius, the lower the force required to penetrate the human skin. Thus, improving the replication process to further decrease the tip radius of the polymer microneedles is beneficial. Implementing a variothermal heating strategy is a possible way to achieve this [24]. The temperature of the mould is then no longer kept constant, but is varied between a high value during the filling stage and a lower value during the cooling stage. Therefore, the formation of a frozen layer can be delayed, which in turn leads to a better and more uniform filling of micro features [10]. 
(a) 150 layers:

hole length $=1004 \pm 26 \mu \mathrm{m}$

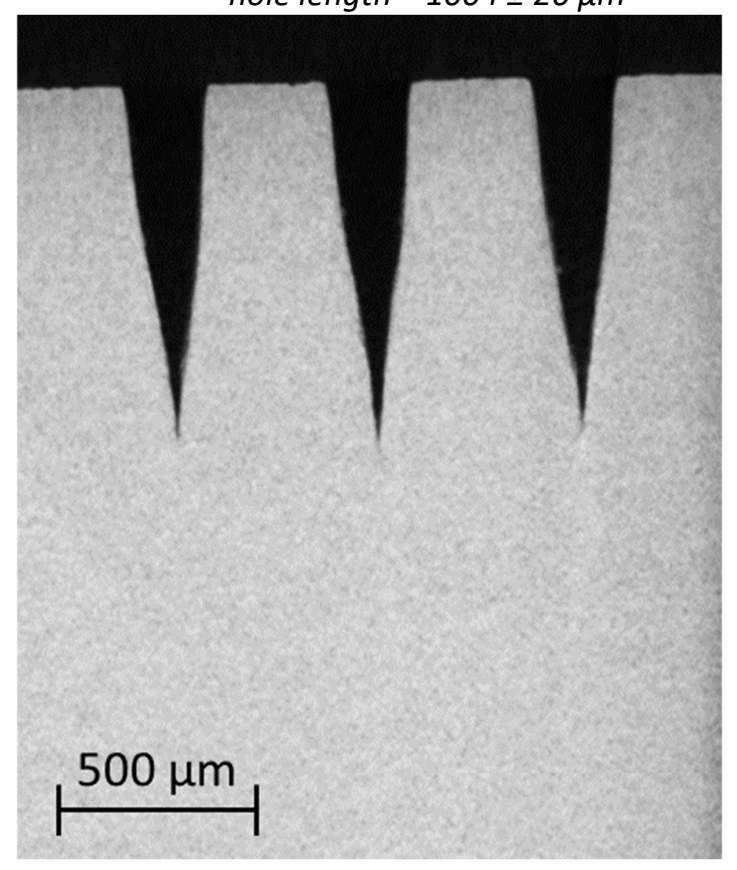

(c) 450 layers:

hole length $=1653 \pm 21 \mu \mathrm{m}$

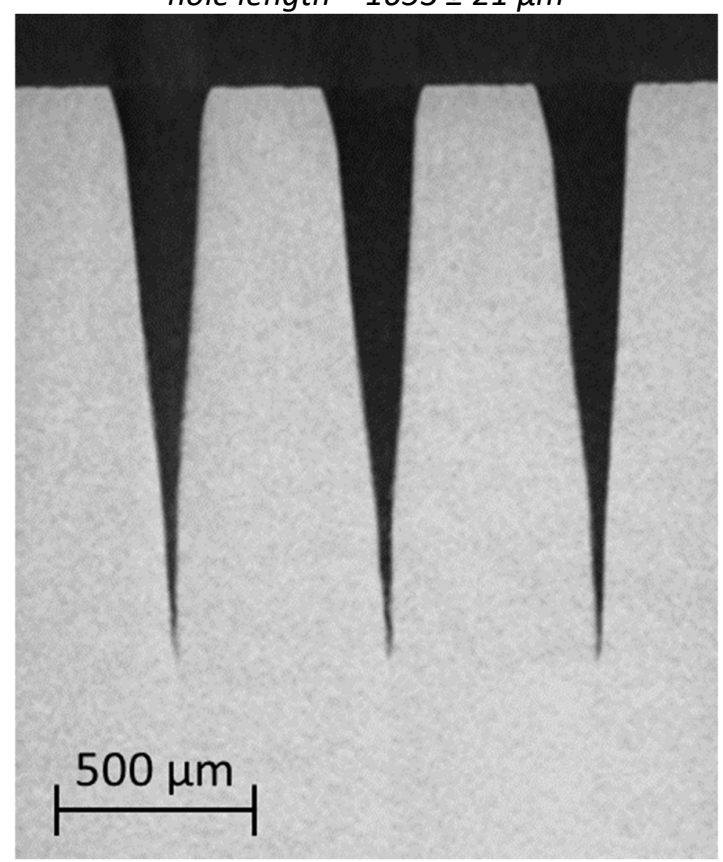

(b) 300 layers:

hole length $=1376 \pm 28 \mu \mathrm{m}$

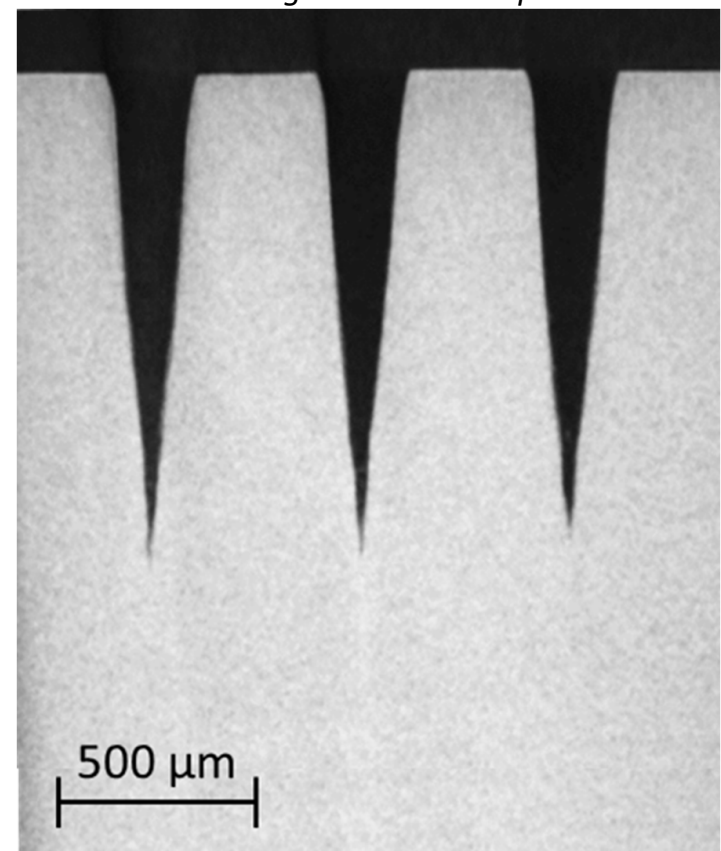

(d) 600 layers:

hole length $=1803 \pm 21 \mu \mathrm{m}$

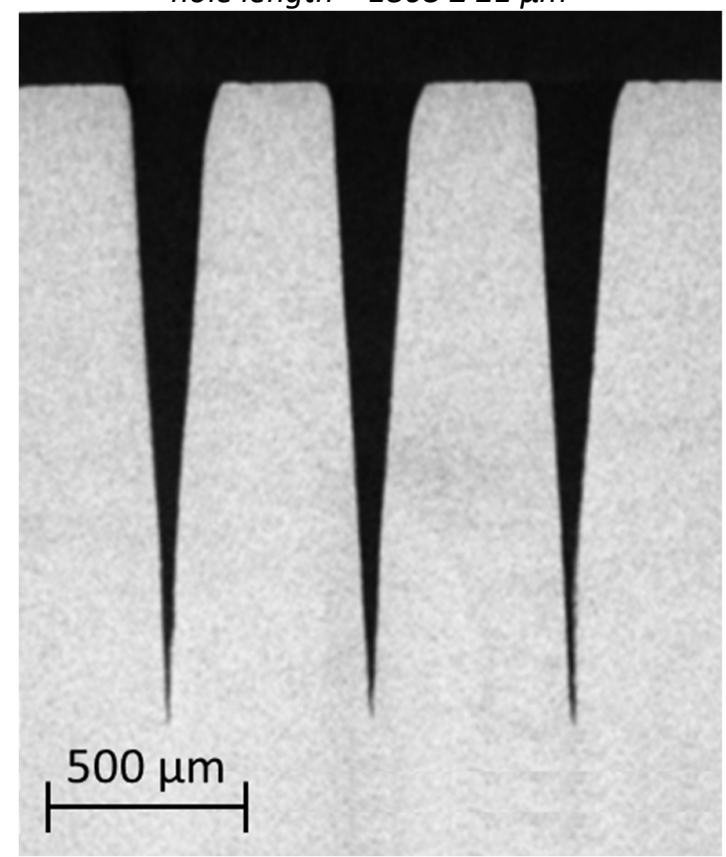

FIGURE 3 Reconstructed $\mu \mathrm{CT} X Y$ datasets for the four different laser parameter settings: (a) 150 layers; (b) 300 layers; (c) 450 layers and (d) 600 layers. 
(a) 150 layers:

needle length $=912 \pm 19 \mu \mathrm{m}$ tip radii $=10.3 \pm 3.4 \mu \mathrm{m}$

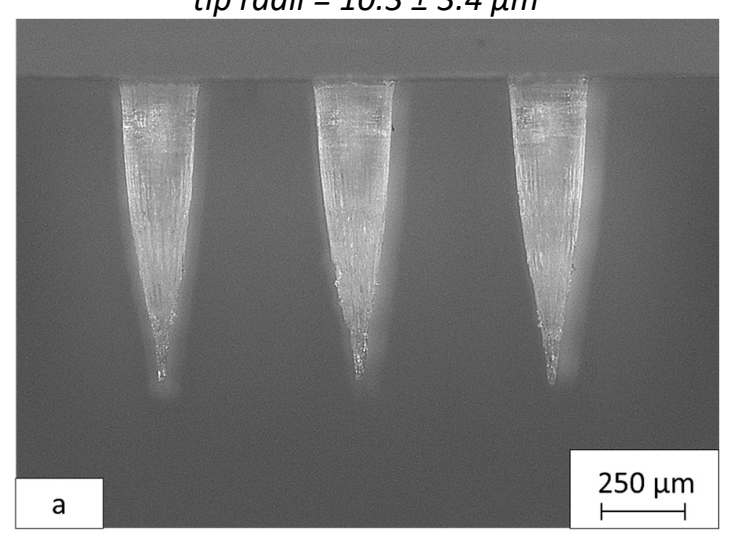

(c) 450 layers:

needle length $=1259 \pm 14 \mu \mathrm{m}$

tip radii $=17.3 \pm 3.4 \mu \mathrm{m}$

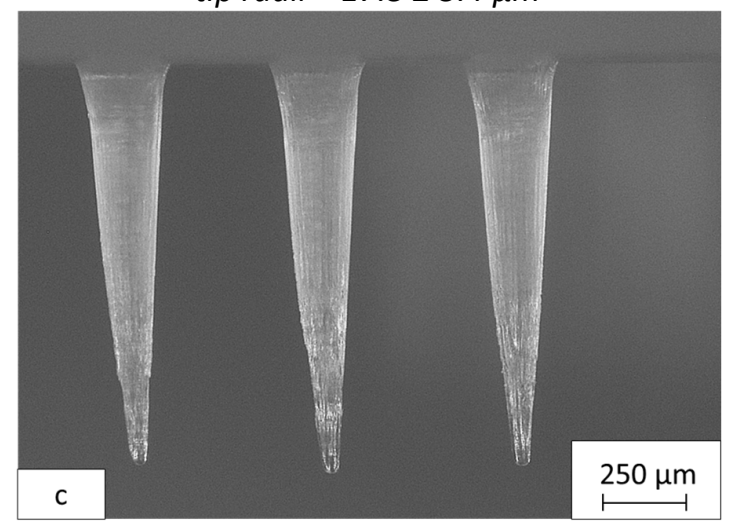

(b) 300 layers:

needle length $=1136 \pm 14 \mu \mathrm{m}$ tip radii $=14.2 \pm 2.5 \mu \mathrm{m}$

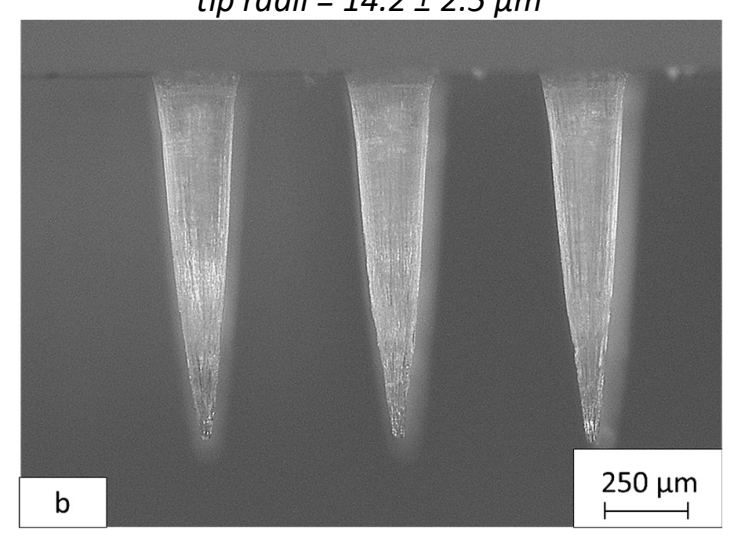

(d) 600 layers:

needle length $=1307 \pm 14 \mu \mathrm{m}$ tip radii $=19.6 \pm 3.6 \mu \mathrm{m}$

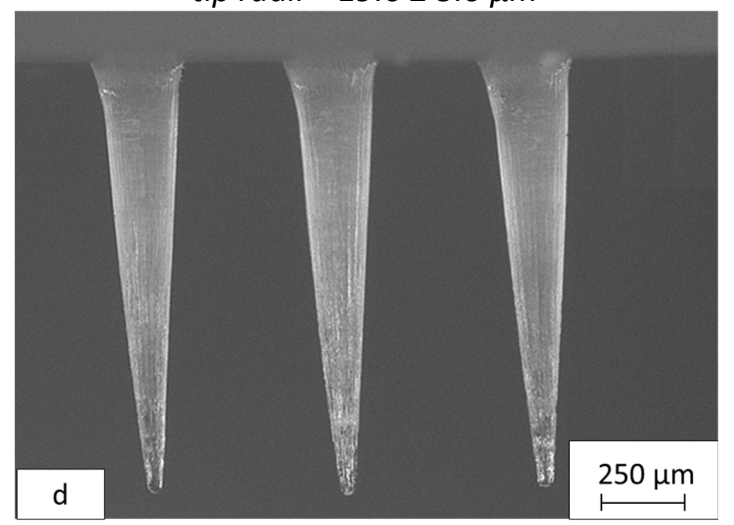

FIGURE 4 Microscopic images of the polymer microneedles, achieved by replicating the laser ablated micro holes with four different laser settings: (a) 150 layers; (b) 300 layers; (c) 450 layers and (d) 600 layers. 


\section{CONCLUSIONS}

In this study, a method to produce solid polymer microneedles using laser ablated moulds in an injection moulding process was successfully demonstrated. Cone-shaped micro holes with very small tip radii were ablated in a tool steel insert. By changing laser process parameters, the height of the micro holes could be varied. After replicating the micro-holes, thermoplastic solid microneedles were achieved with tip radii $<20 \mu \mathrm{m}$. The replication fidelity was volumetrically assessed and the deeper the micro holes, the lower the replication (ranging from $97 \%$ - 85\%).

The major advantages of the proposed laser ablation technique to create cone-shaped micro holes are the very sharp tip radii, the fast and flexible manufacturing and the low cost. Thus, the investigated process chain is proven to be a suitable mass manufacturing method to produce solid polymer microneedles with small tip-radii.

\section{ACKNOWLEDGEMENTS}

The authors would like to thank Dr Jeroen Soete for his guidance in the X-ray computed tomography imaging of the samples at the KU Leuven facilities.

\section{FUNDING}

This work was supported by internal funding from KU Leuven Campus Diepenbeek. 


\section{REFERENCES}

[1] K. Ita, "Transdermal delivery of drugs with microneedles-potential and challenges," Pharmaceutics, vol. 7, no. 3, pp. 90-105, 2015.

[2] H. Juster, B. van der Aar, and H. de Brouwer, "A review on microfabrication of thermoplastic polymer-based microneedle arrays," Polym. Eng. Sci., vol. 59, no. 5, pp. 877-890, 2019.

[3] R. F. Donnelly, T. R. R. Singh, D. I. J. Morrow, and A. D. Woolfson, Microneedle-mediated Transdermal and Intradermal Drug Delivery. Pondicherry, 2012.

[4] R. F. Donnelly, T. R. R. Singh, and A. D. Woolfson, "Microneedle-based drug delivery systems: Microfabrication, drug delivery, and safety," Drug Deliv., vol. 17, no. 4, pp. 187-207, May 2010.

[5] R. F. Donnelly, T. R. R. Singh, E. Larrañeta, and M. T. C. McCrudden, Microneedles for Drug and Vaccine Delivery and Patient Monitoring. Chichester, 2018.

[6] J. W. Lee, M. R. Han, and J. H. Park, "Polymer microneedles for transdermal drug delivery," J. Drug Target., vol. 21, no. 3, pp. 211-223, 2013.

[7] S. Indermun et al., "Current advances in the fabrication of microneedles for transdermal delivery," J. Control. Release, vol. 185, no. 1, pp. 130-138, 2014.

[8] R. F. Donnelly et al., "Hydrogel-forming microneedle arrays for enhanced transdermal drug delivery," Adv. Funct. Mater., vol. 22, no. 23, pp. 4879-4890, 2012.

[9] E. Larrañeta, R. E. M. Lutton, a D. Woolfson, and R. F. Donnelly, "Microneedle arrays as transdermal and intradermal drug delivery systems: Materials science, manufacture and commercial development," Mater. Sci. Eng. R Reports, vol. 104, pp. 1-32, Jun. 2016.

[10] G. Tosello, Micro Injection Molding. München: Carl Hanser Verlag GmbH \& Co. KG, 2018.

[11] C. T. Uppuluri et al., "Microneedle-assisted transdermal delivery of Zolmitriptan: effect of microneedle geometry, in vitro permeation experiments, scaling analyses and numerical simulations," Drug Dev. Ind. Pharm., vol. 43, no. 8, pp. 1292-1303, 2017.

[12] P. Janphuang et al., "Polymer based microneedle patch fabricated using microinjection moulding," MATEC Web Conf., vol. 192, p. 01039, 2018.

[13] S. Sharma, A. Saeed, C. Johnson, N. Gadegaard, and A. E. Cass, "Rapid, low cost prototyping of 
transdermal devices for personal healthcare monitoring," Sens. Bio-Sensing Res., vol. 13, pp. 104-108, 2017.

[14] K. J. NAIR, "PhD thesis, Micro-Injection Moulded Microneedles For Drug Delivery," University of Bradford, 2014.

[15] M. J. Kim et al., "Fabrication of Circular Obelisk-Type Multilayer Microneedles Using MicroMilling and Spray Deposition," Front. Bioeng. Biotechnol., vol. 6, no. May, pp. 1-13, 2018.

[16] S. Gao, Z. Qiu, Z. Ma, and Y. Yang, "Development of high efficiency infrared-heating-assisted micro-injection molding for fabricating micro-needle array," Int. J. Adv. Manuf. Technol., vol. 92, no. 1-4, pp. 831-838, 2017.

[17] Jordan David Hamilton, "Master's thesis, Fabrication and Analysis of Injection Molded Plastic Microneedle Arrays," Georgia Institute of Technology, 2010.

[18] K. J. Krieger, N. Bertollo, M. Dangol, J. T. Sheridan, M. M. Lowery, and E. D. O'Cearbhaill, "Simple and customizable method for fabrication of high-aspect ratio microneedle molds using low-cost 3D printing," Microsystems Nanoeng., vol. 5, no. 1, 2019.

[19] T. Henson, "Master's thesis, Replicatie van lasergetextureerde micronaalden met behulp van een kunststof spuitgietproces," KU Leuven, 2019.

[20] V. Goodship, Practical guide to injection moulding, vol. 52, no. 6. Shrewsbury: Rapra Technology Limited, 1997.

[21] A. V Bulgakov and N. M. Bulgakova, "Thermal model of pulsed laser ablation under the conditions of formation and heating of a radiation-absorbing plasma," Quantum Electron., vol. 29, no. 5, pp. 433-437, May 1999.

[22] P. Petkov, S. Scholz, and S. Dimov, "Strategies for material removal in laser milling," in Conference on Multi-Material Micro Manufacture, 2008, vol. 3, no. 4.

[23] A. P. Markopoulos, P. Koralli, G. Kyriakakis, M. Kompitsas, and D. E. Manolakos, "Molecular dynamics simulation of material removal with the use of laser beam," in Materials Forming and Machining, Elsevier, 2016, pp. 117-153.

[24] C. Rytka, P. M. Kristiansen, and A. Neyer, "Iso- and variothermal injection compression moulding of polymer micro- and nanostructures for optical and medical applications," J. Micromechanics Microengineering, vol. 25, no. 6, pp. 1-16, 2015. 
GRAPHICAL ABSTRACT
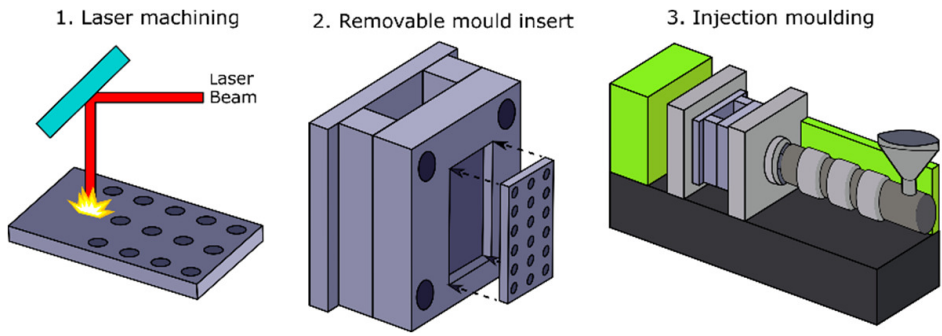

4. Polymer microneedles

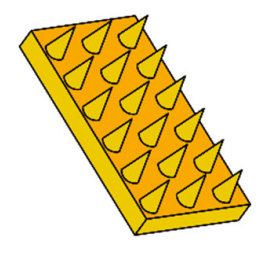

